\title{
Mobility Management with the Central-based Location Area Policy
}

\author{
Ren-Huang Liou, Yi-Bing Lin* \\ Department of Computer Science, National Chiao Tung University, 1001 University \\ Road, Hsinchu, Taiwan 300, ROC
}

\begin{abstract}
In Long Term Evolution (LTE), the cells (the radio coverages of base stations) are grouped into the Tracking Areas (TAs), and the TAs are further grouped into the TA List (TAL). The location of the User Equipment (UE) is tracked at the TAL level. To better capture the "movement locality" of the UE, when the UE leaves the current TAL, the UE is assigned a new TAL whose central TA is the TA where the UE currently resides. This paper investigates the performance of the central-based LTE mobility management scheme, and compares this scheme with the previously proposed central-based mobility management schemes: the movement-based and the distance-based schemes. Our study indicates that under some traffic/mobility patterns, the LTE scheme yields the best performance.
\end{abstract}

Keywords: Location update, mobility management, paging

\footnotetext{
*Corresponding author. Tel.: +886-3-573-1842; fax: +886-3-572-4176.

Email addresses: rhliou@cs.nctu.edu.tw (Ren-Huang Liou), liny@cs.nctu.edu.tw (Yi-Bing Lin)
} 


\section{Introduction}

In most commercial third-generation (3G) mobile telecom networks, the cells (the radio coverages of base stations) are grouped into Location Areas (LAs) [1]. When the User Equipment (UE) moves from an old LA to a new LA, the UE executes the location update procedure to inform the network of its new location. When an incoming call arrives, the network searches the UE by broadcasting the paging messages to all cells in the LA simultaneously.

The 3G LA-based mobility management scheme has several disadvantages $[2,3]$. First, the UE may perform many location updates when the UE resides in the boundary cell of the LA and frequently moves back and forth between two LAs (i.e., the ping-pong effect [4]). Second, when an incoming call arrives, if the LA contains a large number of cells, it may incur large paging traffic to search the UE.

The disadvantages of the LA-based scheme can be avoided in Long Term Evolution (LTE). We first describe the LTE mobility management and then show how the aforementioned issues of the LA-based scheme can be resolved in LTE. In LTE, the cells (Figure 1 (a)) are partitioned into non-overlapped Tracking Areas (TAs; Figure 1 (b)) [5,6]. Every TA has a unique TA Identity (TAI). The TAs are further grouped into TA Lists (TALs). In Figure 1 (c), TAL 1 includes TA 1, TA 2 and TA 3. The Mobility Management Entity (MME; Figure $1(\mathrm{~d})$ ) is responsible for assigning the TAL to the UE through the location update procedure. To mitigate the ping-pong effect, we consider the central policy [5] that assigns the TAL where the UE resides in the central TA of this TAL. In Figure 1 (1), when the UE performs the location update in TA 2, the MME assigns TAL 1 to the UE, where TAL $1=\{\mathrm{TA} 1$, TA 2, TA 


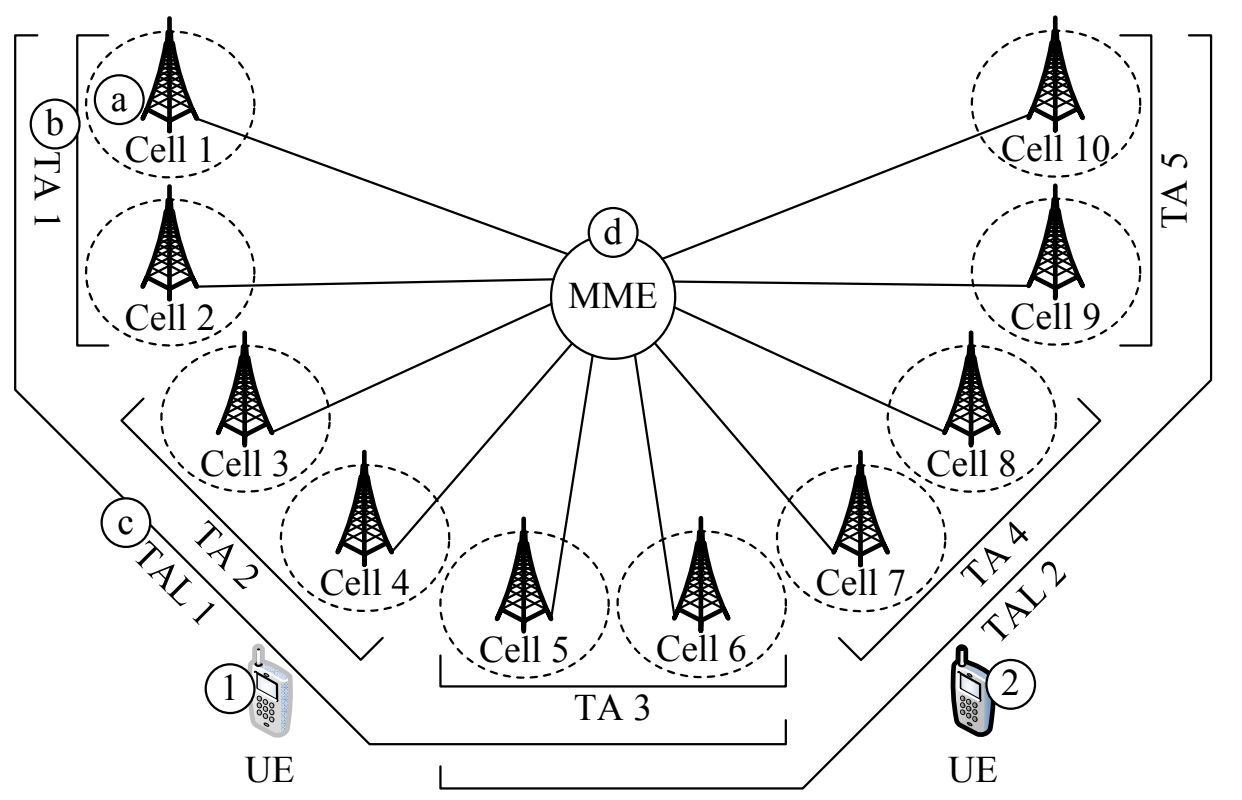

Figure 1: LTE Mobility Management Architecture

$3\}$ centered at TA 2 . Every base station periodically broadcasts its TAI. The UE detects the location change by searching its TAL for the broadcast TAI. If the broadcast TAI is found in its TAL, it means that the UE still resides in its TAL. Otherwise, the UE executes the location update procedure to inform the MME that it has left the current TAL. In Figure 1, when the UE moves from Cell 4 (Figure 1 (1)) to Cell 7 (Figure 1 (2)), the broadcast TA 4 identity is not found in TAL 1, and the UE executes the location update procedure to inform the MME that the UE has moved out of TAL 1 . Then the MME allocates a new TAL to the UE. In Figure 1, the allocated TAL is TAL $2=\{$ TA 3, TA 4 , TA 5$\}$. Note that the TAL is assigned on a peruser basis (i.e., the different UEs may have different TALs), and the newly assigned TAL may overlap with the previously assigned TAL. In Figure 1, 
TA 3 is included in both TAL 1 and TAL 2. We also note that the $3 \mathrm{G}$ LA-based location update is a special case of the LTE TAL-based location update when the size of an LA is equal to that of a TAL and the TAL only contains one TA. When an incoming call arrives, the cells of the UE's TAL will page the UE. In most commercial $3 \mathrm{G}$ mobile networks, all cells in the LA of the UE will execute the paging procedure simultaneously. To reduce the paging traffic, we consider a LTE paging scheme [7] where the MME records the cell where the UE has interacted with the network (e.g., makes call, receives call, or performs location update). This cell is referred to as the interacted cell. When an incoming call arrives, the MME conducts the TAL paging procedure as follows:

Step 1.1. The MME sends the paging message to the last interacted cell. If the MME receives the paging response from the UE, the MME establishes the connection to the UE to deliver the incoming call, and the paging procedure is terminated. Otherwise (i.e., the MME does not receive the response within a timeout period), Step 1.2 is executed.

Step 1.2. The MME sends the paging messages to the TA of the last interacted cell. If the MME receives the paging response, the connection is established, and the paging procedure is terminated. Otherwise, Step 1.3 is executed.

Step 1.3. The MME sends the paging messages to all cells in the TAL to search the UE. After the UE sends the paging response to the MME, the incoming call is delivered to the UE.

We define a polling cycle as a period between when the MME sends the 
paging messages to the cells and when the MME receives the paging response or a timeout occurs. Let $N_{p}$ be the maximum number of the polling cycles before the UE is found. Steps 1.1-1.3 indicate that $N_{p}=3$ in the TAL paging scheme.

Our previous work has proposed an analytic model to compare the performance between the central-based LTE mobility management and the $3 \mathrm{G}$ mobility management, which shows that the LTE mobility management outperforms the 3G mobility management [7]. This paper describes a simulation model for the TAL-based scheme, and compares the TAL-based scheme with the movement-based $[2,8]$ and the distance-based $[8,9,10,13]$ schemes with the Shortest-Distance-First (SDF) paging $[2,10]$. Note that the movementbased and the distance-based schemes with the SDF paging were intensively studied in the literature. However, these schemes have not been exercised in any commercial mobile telecom network because their implementations are not feasible (to be elaborated). The central policy and the TAL paging scheme we described for the LTE mobility management partially implement the distance-based scheme with the SDF paging, and we will show that our approach can capture the advantages of the distance-based scheme with the SDF paging.

This paper is organized as follows. Section 2 introduces the TAL-based, the movement-based, and the distance-based location updates. Then we describe the SDF paging scheme. Section 3 proposes a simulation model for these location update and paging schemes. Section 4 compares the performance of the TAL-based, the movement-based, and the distance-based schemes by numerical examples, and the conclusions are given in Section 5 . 


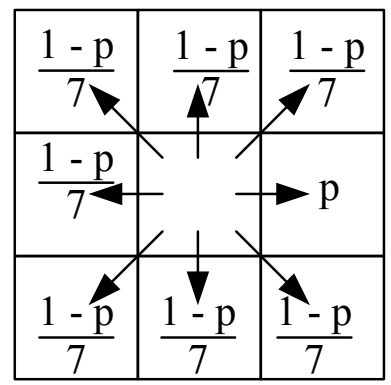

Figure 2: Mesh Cell Configuration and the Movement Directions

\section{Location Update and Paging Schemes}

This section describes the TAL-based, the movement-based, and the distance-based location update schemes. For the description purpose, we consider a two-dimensional (2-D) mesh cell configuration (i.e., Manhattanstreet layout) as illustrated in Figure 2, where a rectangular represents a cell. In this configuration, each cell has eight neighboring cells. Many previous studies assume that the UE moves to one of the neighboring cells with the same routing probability $[2,8,10]$. We relax this assumption to accommodate heterogeneous routing patterns. In our random walk model, the UE moves to the right-hand side neighboring cell with routing probability $p$, and moves to one of other neighboring cells with the same probability $(1-p) / 7$. If $p=0.125$, then the routing pattern is homogeneous (i.e., the UE movement exhibits locality; e.g., a pedestrian or a vehicle in local roads), which is the same as those in $[2,8,10]$. If $p=1$, the UE moves to one direction (e.g., a vehicle in highways). Note that in [7], we used the 1-D model to represent the UE movement in highways. On the other hand, this paper uses 2-D model for city layout. We have showed that the effects of the input parameters in 1-D and 2-D models are similar [7]. Based on the 2-D cell configuration, we 


\begin{tabular}{|c|c|c|c|c|c|c|c|c|c|c|c|}
\hline 1,12 & $\begin{array}{r}2,12 \\
\text { ГA } 1,\end{array}$ & 3,12 & 4,12 & $\begin{array}{l}5,12 \\
\text { TA 2, }\end{array}$ & 6,12 & \begin{tabular}{|l}
7,12 \\
\end{tabular} & \multicolumn{2}{|c|}{ TA 3,4 } & \multirow{2}{*}{\multicolumn{3}{|c|}{$\begin{array}{r}10,12|11,12| 12,12 \\
\mathbf{T A} 4, \mathbf{4} \\
10,11|11,11| 12,11\end{array}$}} \\
\hline 1,11 & 2,11 & $\mid 3,11$ & 4,11 & $\mid 5,11$ & 6,11 & 7,11 & 8,11 & 9,11 & & & \\
\hline 1,10 & 2,10 & 3,10 & 4,10 & 5,10 & 6,10 & 7,10 & 8,10 & 9,10 & 10,10 & 11,10 & 12,10 \\
\hline 1,9 & 2,9 & 3,9 & 4,9 & 5,9 & 6,9 & 7,9 & 8,9 & 9,9 & 10,9 & $\mid 11,9$ & 12,9 \\
\hline & TA 1, & & -7 & TA 2 , & & & TA 3, & $3-$ & - & ГA 4,3 & 3 \\
\hline 1,8 & 2,8 & 3,8 & 4,8 & 5,8 & 6,8 & 7,8 & 8,8 & 9,8 & 10,8 & $|11,8|$ & 12,8 \\
\hline 1,7 & 2,7 & 3,7 & 4,7 & 5,7 & 6,7 & 7,7 & 8,7 & 9,7 & 10,7 & 11,7 & 12,7 \\
\hline 1,6 & 2,6 & 3,6 & 4,6 & 5,6 & 6,6 & 7,6 & 8,6 & 9,6 & 10,6 & $|11,6|$ & 12,6 \\
\hline- & TA 1, & 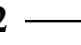 & -7 & TA 2, & $\overline{-}$ & -7 & TA 3, & $2-$ & $\tau^{\prime}$ & TA 4,2 & 2 \\
\hline 1,5 & 2,5 & 3,5 & 4,5 & 5,5 & 6,5 & 7,5 & 8,5 & 9,5 & 10,5 & 11,5 & 12,5 \\
\hline 1,4 & 2,4 & 3,4 & 4,4 & 5,4 & 6,4 & 7,4 & 8,4 & 9,4 & 10,4 & 11,4 & 12,4 \\
\hline 1,3 & 2,3 & 3,3 & 4,3 & 5,3 & 6,3 & 7,3 & 8,3 & 9,3 & 10,3 & 11,3 & 12,3 \\
\hline & TA $\mathbf{1}$, & & 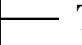 & TA 2, & - & -7 & ГА 3, & 1 & - & TA 4, & $1-$ \\
\hline 1,2 & 2,2 & 3,2 & 4,2 & 5,2 & 6,2 & 7,2 & 8,2 & 9,2 & 10,2 & $\mid 11,2$ & $\mid 12,2$ \\
\hline 1,1 & 2,1 & 3,1 & 4,1 & 5,1 & 6,1 & 7,1 & 8,1 & 9,1 & 10,1 & 11,1 & 12,1 \\
\hline
\end{tabular}

Figure 3: The TAL Configuration $\left(N_{C}=N_{T}=9\right)$

describe the location updates and the Shortest-Distance-First (SDF) paging in the following subsections.

\subsection{TAL-based Location Update}

Let $N_{C}$ be the number of cells in a TA and $N_{T}$ be the number of TAs in a TAL. Figure 3 illustrates a TAL configuration, where $N_{C}=N_{T}=9$. In the TAL, a cell is labelled as $\langle x, y\rangle$, where $x$ is the column label, $y$ is 
the row label, and $1 \leq x, y \leq \sqrt{N_{C} N_{T}}$. Similarly, a TA is labelled as $\langle X, Y\rangle$, where $1 \leq X, Y \leq \sqrt{N_{T}}$. TA $\langle X, Y\rangle$ includes cells $\langle x, y\rangle$ where $(X-1) \sqrt{N_{C}}+1 \leq x \leq X \sqrt{N_{C}}$ and $(Y-1) \sqrt{N_{C}}+1 \leq y \leq Y \sqrt{N_{C}}$. To simplify our discussion on the central policy mentioned in Section 1, we assume that $\sqrt{N_{T}}$ is an odd number. Due to the central policy, when the UE moves out of the current TAL (i.e., the UE moves to Cell $\langle x, y\rangle$ where $x=0$ or $\sqrt{N_{C} N_{T}}+1$, or $y=0$ or $\sqrt{N_{C} N_{T}}+1$ ), the entrance TA is reset to the central TA (i.e., TA $\left\langle\left\lceil\sqrt{N_{T}} / 2\right\rceil,\left\lceil\sqrt{N_{T}} / 2\right\rceil\right\rangle$ ), and the entrance cell is reset to the cell in TA $\left\langle\left\lceil\sqrt{N_{T}} / 2\right\rceil,\left\lceil\sqrt{N_{T}} / 2\right\rceil\right\rangle$ at the same relative position. For example, in Figure 3, when the UE moves from Cell $\langle 9,5\rangle$ to Cell $\langle 10,5\rangle$, Cell $\langle 10,5\rangle$ is reset to Cell $\langle 4,5\rangle$. Cell $\langle 10,5\rangle$ in TA $\langle 4,2\rangle$ is at the same relative position as Cell $\langle 4,5\rangle$ in TA $\langle 2,2\rangle$.

\subsection{Movement-based and Distance-based Location Updates}

In the movement-based location update, the UE maintains a counter to record the number of cell boundary crossings since the last interaction with the network (location update or incoming call). When the counter value reaches the predefined threshold $K$, the UE updates its new location and resets the counter value to zero. Also, when an incoming call is delivered to the UE, the counter value is reset to zero [2].

In the distance-based location update, the UE updates its location when the distance (in terms of the number of cells) between the last interacted cell and the current cell of the UE is $K$. Note that in the distance-based location update, the UE is required to have the knowledge of the cell topology information (i.e., the distance relationship between cells) [3, 8, 11], which can not be practically implemented in a real network. Therefore, the distance- 
based location update is an idealized mechanism, and is considered in this paper for comparison purpose.

The movement-based and the distance-based location updates ensure that the maximum distance between the last interacted cell and the cell where the UE resides is $K-1$ [2]. Define the residing area of the UE as the area which is within the maximum distance $K-1$ from the last interacted cell. Figure 4 illustrates a residing area with $K=5$. If the last interacted cell is Cell $\langle K, K\rangle$ (i.e., Cell $\langle 5,5\rangle$ ), then the residing area of the UE ranges from Cell $\langle 1,1\rangle$ to Cell $\langle 2 K-1,2 K-1\rangle$ (i.e., from Cell $\langle 1,1\rangle$ to Cell $\langle 9,9\rangle$ ). To make a fair comparison with the TAL-based location update, we assume that the size of the residing area of the movement-based and the distance-based location updates is $N_{C} N_{T}$ (i.e., $\left.(2 K-1)^{2}=N_{C} N_{T}\right)$.

In the movement-based and the distance-based schemes, when the UE updates its location or an incoming call is delivered to the UE, the cell where the UE currently resides is reset to Cell $\langle K, K\rangle$ (i.e., the center cell of the residing area). On the other hand, the TAL-based location update resets the central TA of the TAL to the TA of the last interacted cell (note that the last interacted cell may not be the center cell of the TAL). We also note that an incoming call in the TAL-based scheme does not reset the central TA to the TA of the last interacted cell.

\subsection{Shortest-Distance-First (SDF) Paging}

The Shortest-Distance-First (SDF) paging proposed in [2] is described as follows. The residing area of the UE (i.e., Cell $\langle 1,1\rangle$ to Cell $\langle 2 K-1,2 K-1\rangle$ ) is partitioned into $N_{T}=\min \left(K, N_{p}\right)$ subareas. Let $A_{j}$ be the subarea $j$ where $0 \leq j \leq N_{T}-1$. Subarea $A_{j}$ includes Cells $\langle x, y\rangle$ where $l_{j} \leq x, y \leq r_{j}$. The 


\begin{tabular}{|c|c|c|c|c|c|c|c|c|}
\hline 1,9 & 2,9 & 3,9 & 4,9 & 5,9 & 6,9 & 7,9 & 8,9 & 9,9 \\
\hline 1,8 & 2,8 & 3,8 & 4,8 & 5,8 & 6,8 & 7,8 & 8,8 & 9,8 \\
\hline 1,7 & 2,7 & 3,7 & 4,7 & 5,7 & 6,7 & 7,7 & 8,7 & 9,7 \\
\hline 1,6 & 2,6 & 3,6 & 4,6 & 5,6 & 6,6 & 7,6 & 8,6 & 9,6 \\
\hline 1,5 & 2,5 & 3,5 & 4,5 & 5,5 & 6,5 & 7,5 & 8,5 & 9,5 \\
\hline 1,4 & 2,4 & 3,4 & 4,4 & 5,4 & 6,4 & 7,4 & 8,4 & 9,4 \\
\hline 1,3 & 2,3 & 3,3 & 4,3 & 5,3 & 6,3 & 7,3 & 8,3 & 9,3 \\
\hline 1,2 & 2,2 & 3,2 & 4,2 & 5,2 & 6,2 & 7,2 & 8,2 & 9,2 \\
\hline 1,1 & 2,1 & 3,1 & 4,1 & 5,1 & 6,1 & 7,1 & 8,1 & 9,1 \\
\hline
\end{tabular}

Figure 4: The Residing Area Layout for the Movement-based and the Distance-based Location Updates with the SDF Paging $\left(K=5\right.$ and $\left.N_{p}=3\right)$

boundary values $l_{j}$ and $r_{j}$ are computed as

$$
l_{j}=K-\left\lfloor\frac{K(j+1)}{N_{T}}\right\rfloor+1 \text { and } r_{j}=K+\left\lfloor\frac{K(j+1)}{N_{T}}\right\rfloor-1
$$

When an incoming call arrives, the network first partitions the residing area of the UE into the subareas according to (1). Then the network searches the subareas $A_{0}, A_{1}, \ldots, A_{N_{T}-1}$ sequentially until the UE is found (where $\left.A_{0} \subseteq A_{1} \subseteq \ldots \subseteq A_{N_{T}-1}\right)$

Figure 4 shows an SDF partitioning example where $K=5$ and $N_{p}=3$. In this figure, $N_{T}=\min \left(K, N_{p}\right)=3, l_{0}=r_{0}=5, l_{1}=3, r_{1}=7, l_{2}=1$, and $r_{2}=9$. Suppose that the UE resides in Cell $\langle 4,5\rangle$. When an incoming 
call arrives, the network identifies the UE's location after searching $A_{0}$ and $A_{1}$. Therefore, the total number of searched cells is 26 (one cell in $A_{0}$ and 25 cells in $\left.A_{1}\right)$.

We note that the SDF paging is never implemented in commercial mobile telecom network because it is difficult to dynamically define the neighboring cells (because the radio coverage may change due to the radio characteristics and the "adjacent cells" also change). Similarly, it is not practical to set $N_{C}=1$ in LTE.

\section{Simulation Model}

We use the discrete event simulation to investigate the performance of the location update and paging schemes described in the previous sections. We consider two performance measures:

- $C_{u, s}$ : the expected number of location updates between two consecutive incoming calls for the " $s$ " scheme, where $s \in\{T, M, D\}$ denoting the TAL-based, the movement-based, and the distance-based location updates, respectively

- $C_{p, s}$ : the expected number of cells that page the UE when an incoming call arrives for the TAL paging $(s=T)$, the movement-based scheme with the SDF paging $(s=M)$, and the distance-based scheme with the SDF paging $(s=D)$, respectively

It is clear that the smaller the above performance measures, the better the performance of the location update and paging schemes. In our simulation model, an event $e$ has two attributes: 
- The type attribute indicates the event type. There are two event types. A Call event represents a call arrival. When this event occurs, the core network establishes the connection to a UE through the paging procedure. A Move event represents that the UE crosses the cell boundary to a neighboring cell.

- The $t s$ attribute indicates the timestamp when the event occurs.

The inter-call arrival time $t_{c}$ is a random number drawn from an exponential generator $G_{C}$ with the mean $1 / \lambda_{c}$. The inter-move time $t_{m}$ is a random number drawn from a Gamma generator $G_{M}$ with the mean $1 / \lambda_{m}$ and the variance $V$. We consider the Gamma distribution because it has been shown that the distribution of any positive random variable can be approximated by a mixture of Gamma distributions [12]. The Gamma distribution was used to model UE movement in many studies $[2,7,10,10]$, and is used in this paper to investigate the impact of variance for cell residence times. The direction of each UE movement is determined by a uniform random number $U$ between 0 and 1 drawn from a generator $G_{D}$. Four labels are used to track a UE's location:

- $\langle\bar{x}, \bar{y}\rangle$ : the column and the row labels of the last interacted cell, respectively

- $\left\langle x^{*}, y^{*}\right\rangle$ : the column and the row labels of the cell where the UE currently resides, respectively

There are three output variables in the simulation model:

- $n_{a}$ : the number of call arrivals 
- $n_{u}$ : the number of location updates

- $n_{p}$ : the number of cells that page the UE when an incoming call arrives

From the above output variables, we compute

$$
C_{u, s}=\frac{n_{u}}{n_{a}} \text { and } C_{p, s}=\frac{n_{p}}{n_{a}}
$$

In the simulation, a clock $t$ is maintained to indicate the simulation progress, which is the timestamp of the event being processed. All events are inserted into the event list, and are deleted/processed from the event list in the non-decreasing timestamp order. Figure 5 illustrates the simulation flow chart for the TAL-based location update and paging with the following steps:

Step 1. Set all output variables (i.e., $n_{a}, n_{u}$ and $n_{p}$ ) and the simulation clock $t$ to 0 . Initialize $\bar{x}, \bar{y}, x^{*}$, and $y^{*}$ to an integer randomly selected between 1 and $\sqrt{N_{C} N_{T}}$.

Step 2. The first Call event $e_{1}$ and Move event $e_{2}$ are generated and are inserted into the event list. For event $e_{1}, e_{1}$.type is Call and $e_{1} \cdot t s=t+t_{c}$ where $t_{c}$ is generated from $G_{C}$. For event $e_{2}, e_{2}$.type is Move and $e_{2} . t s=t+t_{m}$ where $t_{m}$ is generated from $G_{M}$.

Step 3. The first event $e$ in the event list is deleted, and is processed based on its type in Step 4. The clock $t$ is set to e.ts.

Step 4. If e.type is Call, then Step 5 is executed. If e.type is Move, the simulation proceeds to Step 15. 


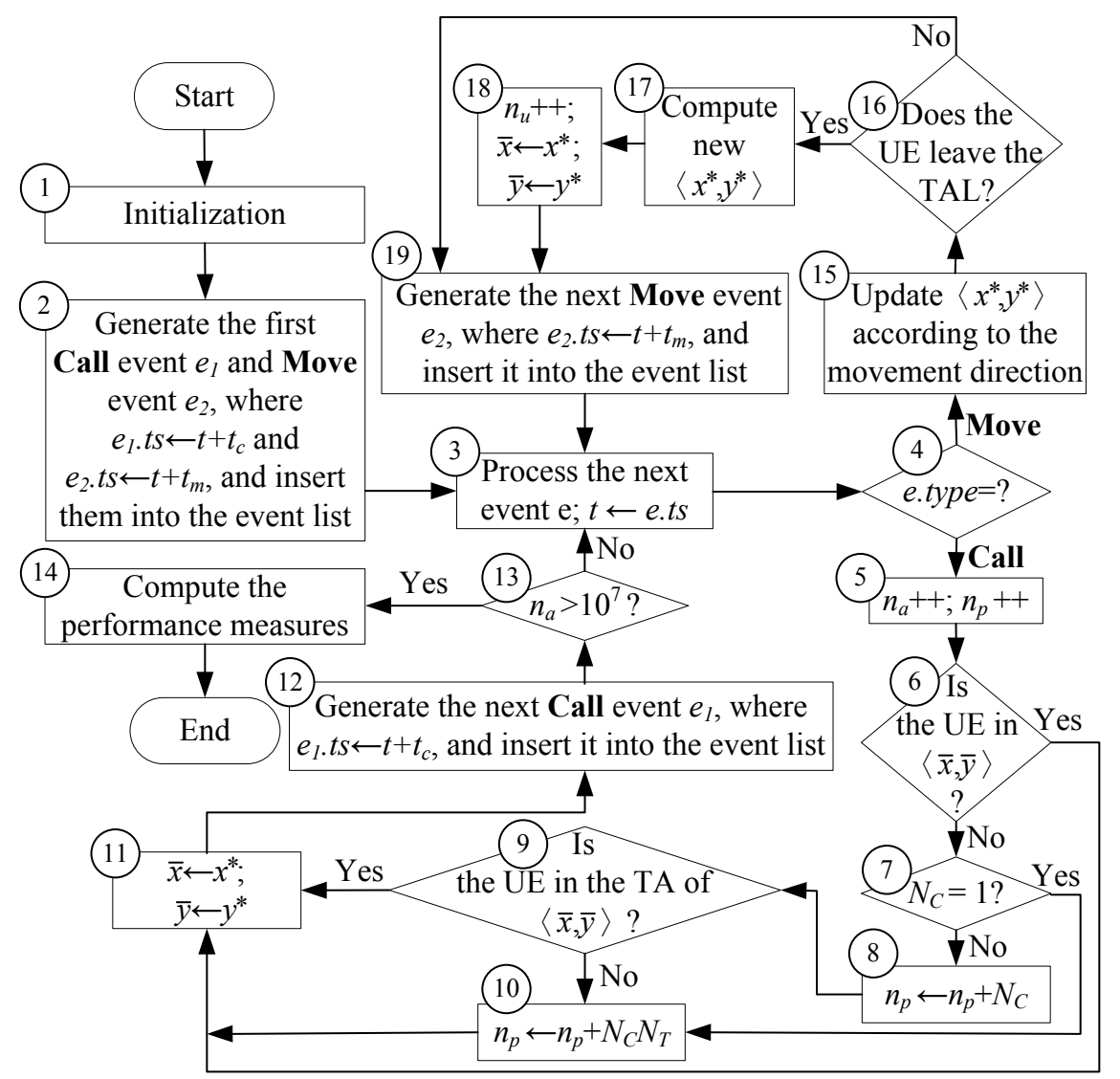

Figure 5: Simulation Flow Chart for TAL-based Location Update and Paging

Step 5. When an incoming call arrives, $n_{a}$ is incremented by one. The last interacted cell pages the UE, and $n_{p}$ is incremented by one.

Step 6. If the UE resides in the last interacted cell (i.e., $\left\langle x^{*}, y^{*}\right\rangle=\langle\bar{x}, \bar{y}\rangle$ ), then Step 11 is executed. Otherwise, the simulation proceeds to Step 7.

Step 7. If $N_{C}=1$ (i.e., the TA only contains one cell), the TA of the last interacted cell has paged the UE in Step 5, and Step 10 is executed. 
Otherwise, Step 8 is executed.

Step 8. All cells in the TA of the last interacted cell page the UE, and $n_{p}$ is incremented by $N_{C}$.

Step 9. If the UE resides in the TA of the last interacted cell (i.e., $\left\lceil x^{*} / \sqrt{N_{C}}\right\rceil$ $=\left\lceil\bar{x} / \sqrt{N_{C}}\right\rceil$ and $\left.\left\lceil y^{*} / \sqrt{N_{C}}\right\rceil=\left\lceil\bar{y} / \sqrt{N_{C}}\right\rceil\right)$, then Step 11 is executed. Otherwise, the simulation proceeds to Step 10.

Step 10. All cells in the TAL page the UE, and $n_{p}$ is incremented by $N_{C} N_{T}$.

Step 11. The MME updates the last interacted cell by setting $\langle\bar{x}, \bar{y}\rangle$ to $\left\langle x^{*}, y^{*}\right\rangle$.

Step 12. The next Call event $e_{1}$ is generated and is inserted into the event list where $e_{1} \cdot t s=t+t_{c}$.

Step 13. If ten million of Call events have been processed, then Step 14 is executed. Otherwise, the simulation proceeds to Step 3. In our experience, ten million of Call events are enough to produce stable statistics.

Step 14. The performance measures are computed according to (2), and the simulation is terminated.

Step 15. If e.type is Move in Step 4, then this step is executed. The movement direction of the UE is determined by the uniform random number $U$ which is generated by $G_{D}$. As shown in Figure 2, new $\left\langle x^{*}, y^{*}\right\rangle$ are 
computed using the following routing rules.

$$
\left\langle x^{*}, y^{*}\right\rangle \leftarrow \begin{cases}\left\langle x^{*}+1, y^{*}+1\right\rangle, & \text { for } 0 \leq U<\frac{1-p}{7} \\ \left\langle x^{*}, y^{*}+1\right\rangle, & \text { for } \frac{1-p}{7} \leq U<\frac{2(1-p)}{7} \\ \left\langle x^{*}-1, y^{*}+1\right\rangle, & \text { for } \frac{2(1-p)}{7} \leq U<\frac{3(1-p)}{7} \\ \left\langle x^{*}-1, y^{*}\right\rangle, & \text { for } \frac{3(1-p)}{7} \leq U<\frac{4(1-p)}{7} \\ \left\langle x^{*}-1, y^{*}-1\right\rangle, & \text { for } \frac{4(1-p)}{7} \leq U<\frac{5(1-p)}{7} \\ \left\langle x^{*}, y^{*}-1\right\rangle, & \text { for } \frac{5(1-p)}{7} \leq U<\frac{6(1-p)}{7} \\ \left\langle x^{*}+1, y^{*}-1\right\rangle, & \text { for } \frac{6(1-p)}{7} \leq U<1-p \\ \left\langle x^{*}+1, y^{*}\right\rangle, & \text { for } 1-p \leq U \leq 1\end{cases}
$$

Step 16. If the UE leaves the current TAL (i.e., $x^{*}=0$ or $\sqrt{N_{C} N_{T}}+1$, or $y^{*}=0$ or $\left.\sqrt{N_{C} N_{T}}+1\right)$, Step 17 is executed. Otherwise, the flow jumps to Step 19.

Step 17. As mentioned in Subsection 2.1, when the UE leaves the current TAL, the entrance cell is reset to the cell in TA $\left\langle\left\lceil\sqrt{N_{T}} / 2\right\rceil,\left\lceil\sqrt{N_{T}} / 2\right\rceil\right\rangle$ in the new TAL. Therefore, $x^{*}$ and $y^{*}$ are set as

$$
x^{*} \leftarrow \sqrt{N_{C}}\left(\left\lceil\frac{\sqrt{N_{T}}}{2}\right\rceil-\left\lceil\frac{x^{*}}{\sqrt{N_{C}}}\right\rceil\right)+x^{*}
$$

and

$$
y^{*} \leftarrow \sqrt{N_{C}}\left(\left\lceil\frac{\sqrt{N_{T}}}{2}\right\rceil-\left\lceil\frac{y^{*}}{\sqrt{N_{C}}}\right\rceil\right)+y^{*}
$$

Step 18. $n_{u}$ is incremented by one. The MME updates the last interacted cell; i.e., $\langle\bar{x}, \bar{y}\rangle$ is set to $\left\langle x^{*}, y^{*}\right\rangle$. 
Step 19. The next Move event $e_{2}$ is generated and inserted into the event list where $e_{2} . t s=t+t_{m}$. The simulation jumps to Step 3 .

The differences between Figure 5 and the simulation flow chart for the movement-based location update with the SDF paging are described as follows. An additional variable $n_{m}$ is needed to record the number of cell crossings after the last interaction with the network, and the labels $\langle\bar{x}, \bar{y}\rangle$ are not needed in the movement-based scheme (because the last interacted cell is always $\langle K, K\rangle$ in the movement-based scheme). In Step 1, $n_{m}$ is reset to zero. In Steps $5-10$, if the UE is found in $i$ th polling cycle, $n_{p}$ is incremented by the summation of the number of cells in $A_{0}, A_{1}, \ldots A_{i-1}$. In Step 11, $\left\langle x^{*}, y^{*}\right\rangle$ are reset to $\langle K, K\rangle$, and $n_{m}$ is reset to zero. In Step $15, n_{m}$ is incremented by one, and $\left\langle x^{*}, y^{*}\right\rangle$ is updated according to (3). In Step 16, if $n_{m}=K$, Step 17 is executed. Otherwise, the simulation goes to Step 19. In Step 17, $\left\langle x^{*}, y^{*}\right\rangle$ is reset to $\langle K, K\rangle$, and $n_{m}$ is reset to zero.

The simulation flow chart for the distance-based scheme with the SDF paging is similar to that for the movement-based scheme with the SDF paging except that the variable $n_{m}$ is not needed in the distance-based scheme. Besides, in Step 16, if $x^{*}=0$ or $2 K$ or $y^{*}=0$ or $2 K$, Step 17 is executed. Otherwise, Step 19 is executed.

We validate the simulation model by the previously proposed analytic models $[2,7,10]$. Simulation experiments show that the discrepancies between the analytic and simulation results are within $1 \%$, and the details are omitted. 


\section{Numerical Examples}

This section compares the performance of the TAL-based, movementbased, and the distance-based schemes by numerical examples. We show the results for the TAL (residing area) size $N_{C} N_{T}=(2 K-1)^{2}=15^{2}$. The results for other $N_{C} N_{T}$ values are similar and are omitted. For the SDF paging, $N_{p}=3$ is considered for two reasons. First, from the simulation model we conducted, the paging cost for $N_{p}=3$ is lowest (or near lowest) for the movement-based and the distance-based schemes (the detailed effect of $N_{p}$ on the paging cost will not be presented here). Second, the TAL paging scheme at most pages three times and can be fairly compared with the SDF paging scheme when $N_{p}=3$. For the TA size, we select $N_{C}=5^{2}$.

\subsection{Analysis of the $C_{u, s}$ Performance}

This subsection investigates the effects of $p, \lambda_{m} / \lambda_{c}$, and $V$ on $C_{u, s}$.

Effects of $p$ : Figure 6 (a) plots $C_{u, s} / C_{u, T}$ against $p$. For the movementbased scheme $(s=M), C_{u, M} / C_{u, T}$ decreases as $p$ increases. When $p$ increases, the UE tends to move to one direction, and large $C_{u, T}$ is expected. On the other hand, $p$ does not affect $C_{u, M}$ because this scheme determines if a location update is performed based on "movement count", and "movement count" is not affected by the movement direction $p$. Therefore, $C_{u, M} / C_{u, T}$ decreases as $p$ increases. On the other hand, the effect of $p$ on $C_{u, D}$ is similar to that on $C_{u, T}$, and Figure 6 (a) shows that $C_{u, D} / C_{u, T}$ increases as $p$ increases.

Effects of $\lambda_{m} / \lambda_{c}$ : From the analytic models in [7], $C_{u, T}=\frac{\lambda_{m}}{\lambda_{c} E[M]}$, which is consistent with the simulation results that when $\lambda_{m} / \lambda_{c}$ increases, 


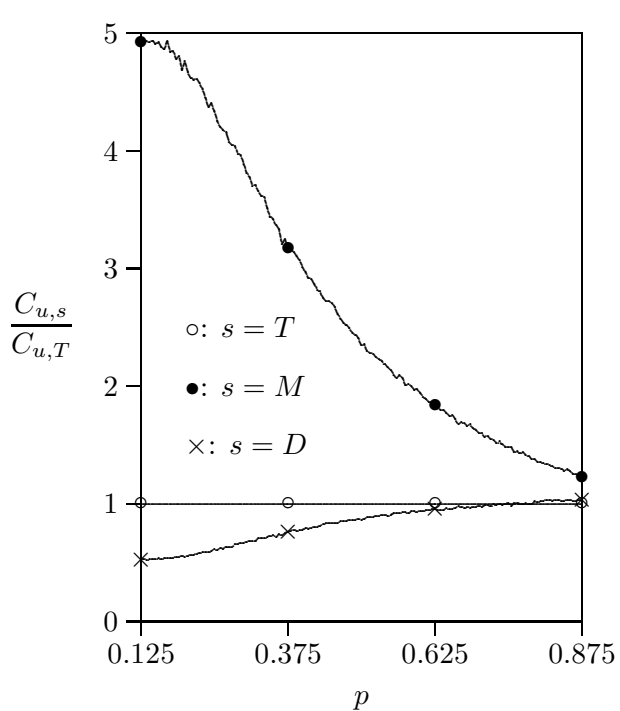

(a) $\lambda_{m} / \lambda_{c}=1$

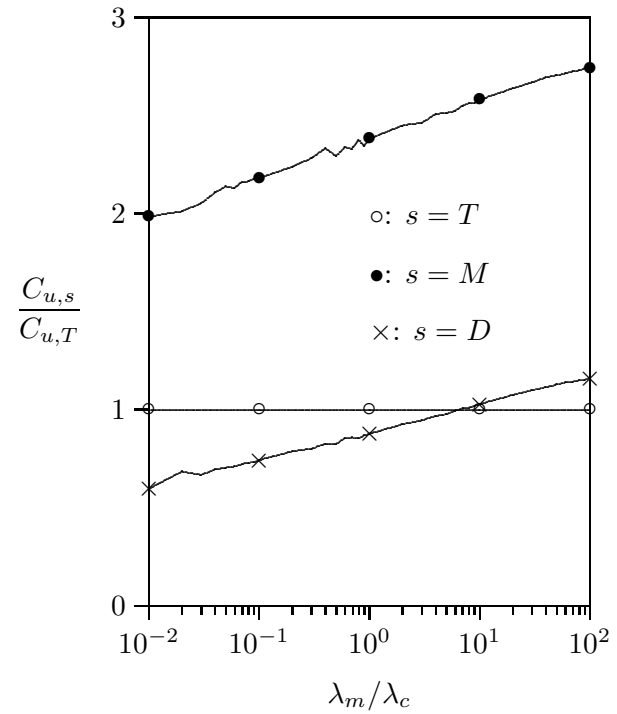

(b) $p=0.5$

Figure 6: Effects of $p$ and $\lambda_{m} / \lambda_{c}$ on $C_{u, s}\left(N_{C}=5^{2}, N_{T}=3^{2}, V=100 / \lambda_{m}^{2}\right.$, and $\left.K=8\right)$

more cell crossings occur in $t_{c}$, and higher $C_{u, s}$ are expected for all schemes. Figure $6(\mathrm{~b})$ shows that $C_{u, M} / C_{u, T}$ and $C_{u, D} / C_{u, T}$ increase as $\lambda_{m} / \lambda_{c}$ increases. For the movement-based and the distance-based schemes, we observe the following fact:

Fact 1. When $K=1$ or $p=1$, the distance-based scheme is the same as the movement-based scheme. For other $K$ and $p$ values, the distance-based scheme always has lower location update cost than the movement-based scheme.

Due to Fact 1 , the values of the $\times$ curves $(s=D)$ are lower than those of the $\bullet$ curves $(s=M)$ in Figures 6 and 7 .

Effects of $V$ : Figure 7 shows that $C_{u, M} / C_{u, T}$ increases as $V$ increases. This 


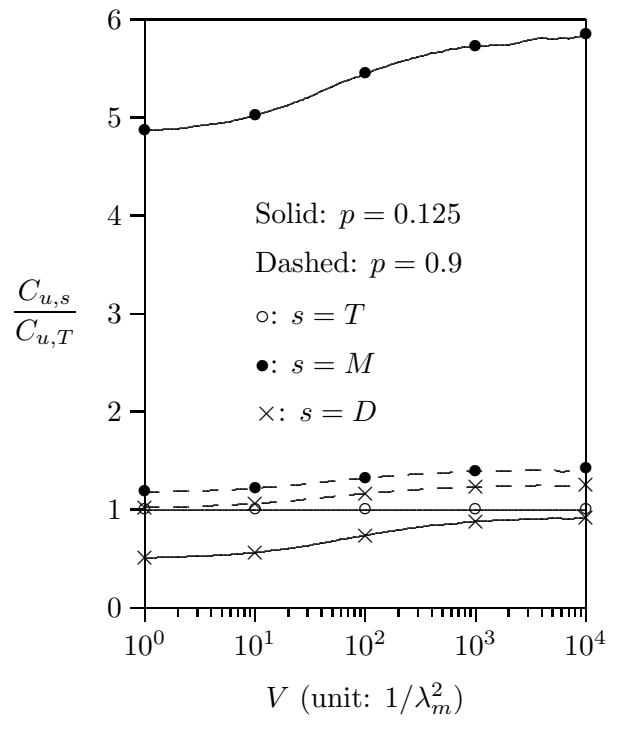

(a) $\lambda_{m} / \lambda_{c}=20$

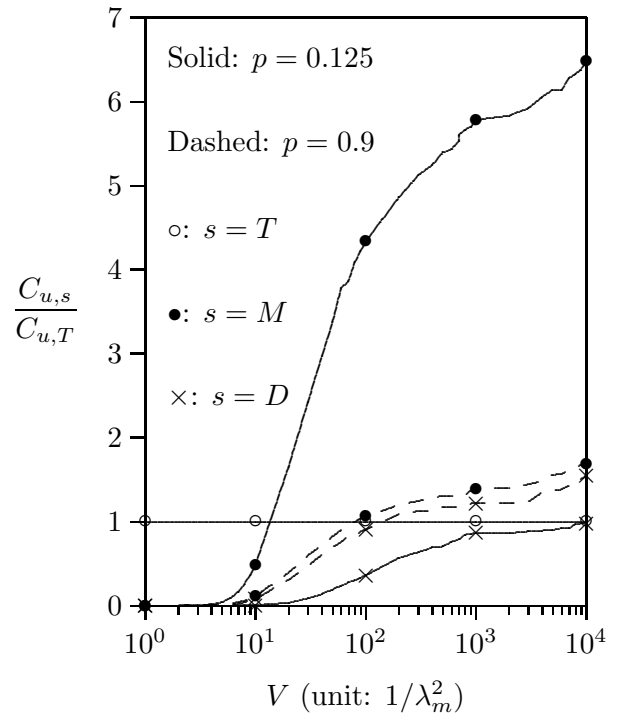

(b) $\lambda_{m} / \lambda_{c}=0.05$

Figure 7: Effects of $V$ and $p$ on $C_{u, s}\left(N_{C}=5^{2}, N_{T}=3^{2}\right.$, and $\left.K=8\right)$

phenomenon is explained as follows. Consider a long observation period $T$. Let $n_{C}$ be the number of the inter-call arrival intervals $t_{c}$ in $T$. Let $n_{M}$ be the number of the cell crossings in $T$. We consider two extreme cases of $V$ (i.e., $V \rightarrow 0$ and $V \rightarrow \infty$ ). When $V \rightarrow 0, t_{m}$ almost have the same fixed value, and the number of the cell crossings in every $t_{c}$ is about a fixed number $n_{M} / n_{C}$. The number of the location updates in every $t_{c}$ for the movement-based scheme is $\left\lfloor n_{M} /\left(n_{C} K\right)\right\rfloor$. Therefore, the number of the location updates in $T$ for $V \rightarrow 0$ is $n_{C}\left\lfloor n_{M} /\left(n_{C} K\right)\right\rfloor$. On the other hand, when $V \rightarrow \infty$, there is one very long $t_{m}$, and other $t_{m} \rightarrow 0$. In this case, all cell crossings occur in a certain $t_{c}$, and the number of the location updates in $T$ for the movement-based scheme is $\left\lfloor n_{M} / K\right\rfloor$. It is clear that $n_{C}\left\lfloor n_{M} /\left(n_{C} K\right)\right\rfloor \leq\left\lfloor n_{M} / K\right\rfloor$ (i.e., $C_{u, M}$ 
increases as $V$ increases). On the other hand, $V$ does not affect $C_{u, T}$ because the incoming call in the TAL-based scheme does not reset the central TA to the TA of the last interacted cell. Therefore, $C_{u, M} / C_{u, T}$ increases as $V$ increases. Similarly, $C_{u, D} / C_{u, T}$ increases as $V$ increases. When $p$ is small, the distance-based scheme outperforms the TAL-based scheme (in Figure 7, the values of the solid $\times$ curve are smaller than 1) because the distance-based scheme always resets the center cell of the residing area to the last interacted cell (i.e., when the UE resides in the center cell of the residing area, the UE is unlikely to leave the residing area for a small $p$, and lower location update cost is expected). On the other hand, when $p$ is large, if $\lambda_{m} / \lambda_{c}$ is large, the TAL-based scheme outperforms the distance-based scheme (in Figure 7 (a), the values of the dashed $\times$ curves are larger than 1 ). This phenomenon can be explained by an extreme case where $p=1$ and $\lambda_{m} / \lambda_{c} \rightarrow \infty$. In this case, the distance-based scheme performs location update for every $K=\left(\sqrt{N_{C} N_{T}}+1\right) / 2$ cell crossings. On the other hand, the TALbased scheme performs location update for every $\sqrt{N_{C}}\left\lceil\sqrt{N_{T}} / 2\right\rceil$ cell crossings. It is clear that $\sqrt{N_{C}}\left\lceil\sqrt{N_{T}} / 2\right\rceil \geq\left(\sqrt{N_{C} N_{T}}+1\right) / 2$. Similarly, when both $p$ and $V$ are large, the TAL-based scheme outperforms the distance-based scheme (in Figure 7 , for $V \geq 200 / \lambda_{m}^{2}$ the values of the dashed $\times$ curves are larger than 1 ). When both $\lambda_{m} / \lambda_{c}$ and $V$ are small, only a small number of cell crossings occur in $t_{c}$ (e.g., less than $K)$, which is unlikely to incur location update for the distance-based scheme. In this case, the distance-based scheme outperforms the TALbased scheme (in Figure 7 (b), for $V \leq 100 / \lambda_{m}^{2}$, the values of the $\times$ 
Table 1: The Mobility Management Scheme with the Lowest Location Update Cost under Different $\lambda_{m} / \lambda_{c}, V$, and $p$ Values

\begin{tabular}{|c|c|c|c|c|c|c|c|}
\hline \multicolumn{4}{|c|}{ Small $\lambda_{m} / \lambda_{c}$} & \multicolumn{4}{c|}{ Large $\lambda_{m} / \lambda_{c}$} \\
\hline \multicolumn{2}{|c|}{ Small $V$} & \multicolumn{2}{|c|}{ Large $V$} & \multicolumn{2}{c|}{ Small $V$} & \multicolumn{2}{c|}{ Large $V$} \\
\hline Small $p$ & Large $p$ & Small $p$ & Large $p$ & Small $p$ & Large $p$ & Small $p$ & Large $p$ \\
\hline Distance & Distance & Distance & TAL & Distance & TAL & Distance & TAL \\
\hline
\end{tabular}

curves are smaller than 1$)$.

From the above discussions, Table 1 lists the mobility management scheme with the lowest location update cost under different input parameter setups. In this table, the TAL-based scheme outperforms the other schemes when (1) $\lambda_{m} / \lambda_{c}$ and $p$ are large or (2) $V$ and $p$ are large.

\subsection{Analysis of the $C_{p, s}$ Performance}

This subsection investigates the effects of $p, \lambda_{m} / \lambda_{c}$, and $V$ on $C_{p, s}$.

Effects of $p$ : It is clear that the paging cost increases as $p$ increases for all schemes. Figure 8 (a) indicates that $C_{p, M} / C_{p, T}$ and $C_{p, D} / C_{p, T}$ increase as $p$ increases.

Effects of $\lambda_{m} / \lambda_{c}$ : When $\lambda_{m} / \lambda_{c}$ increases, the UE is more likely to be far away from the last interacted cell when the next call arrives, and higher $C_{p, s}$ are expected for all $s \in\{T, M, D\}$. Figure 8 (b) shows that $C_{p, M} / C_{p, T}$ and $C_{p, D} / C_{p, T}$ decrease as $\lambda_{m} / \lambda_{c}$ increases. Because the movement-based scheme performs more location updates than the 


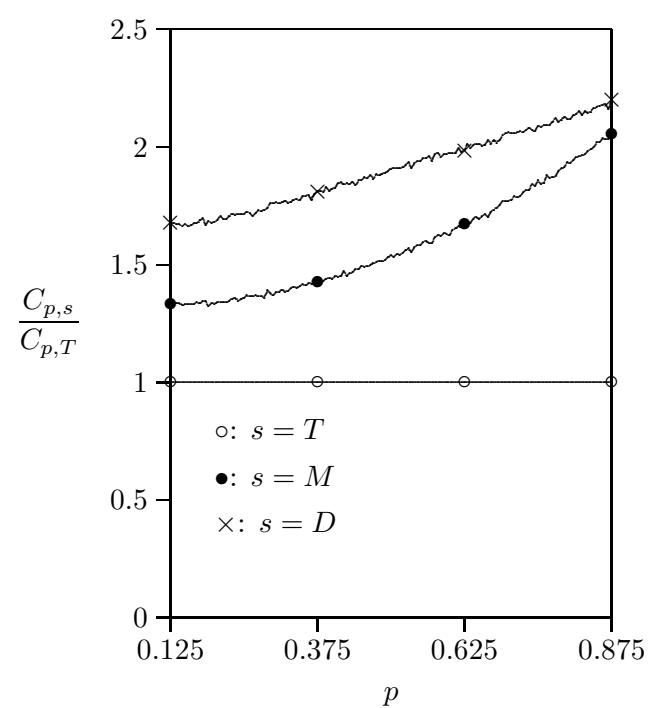

(a) $\lambda_{m} / \lambda_{c}=1$

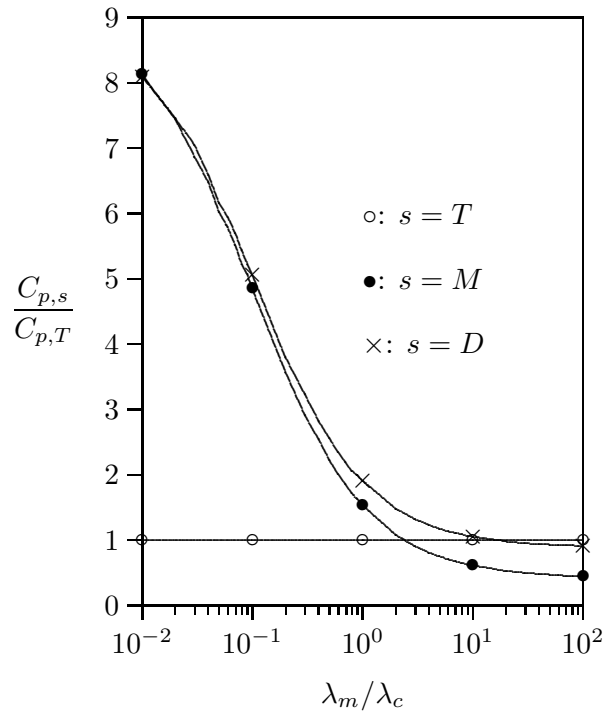

(b) $p=0.5$

Figure 8: Effects of $p$ and $\lambda_{m} / \lambda_{c}$ on $C_{p, s}\left(N_{C}=5^{2}, N_{T}=3^{2}, V=100 / \lambda_{m}^{2}, N_{p}=3\right.$, and $K=8)$

distance-based scheme (Fact 1), the UE is more likely to be found in the last interacted cell for the movement-based scheme, and the movement-based scheme with the SDF paging has lower paging cost than the distance-based scheme with the SDF paging. In Figures 8 and 9 , the values of the $\bullet$ curves $(s=M)$ are lower than those of the $\times$ curves $(s=D)$.

Effects of $V$ : When $V$ is small, most $t_{m}$ have values about $1 / \lambda_{m}$. In this case, if $\lambda_{m} / \lambda_{c}$ is large, the movement-based scheme with the SDF paging outperforms the TAL paging (in Figure 9 (a), for $V \leq 10 / \lambda_{m}^{2}$, the values of the $\bullet$ curves are smaller than 1 ) because the subareas in the SDF paging are symmetric to the last interacted cell (see Figure 4), and 


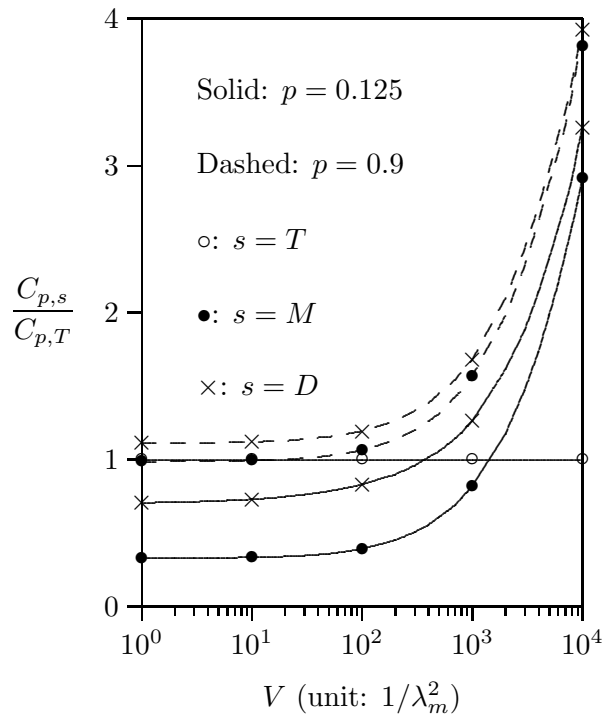

(a) $\lambda_{m} / \lambda_{c}=20$

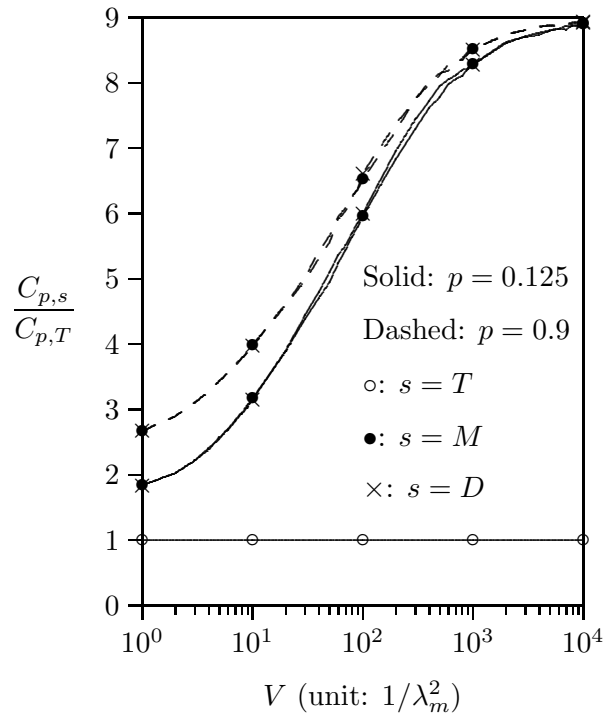

(b) $\lambda_{m} / \lambda_{c}=0.05$

Figure 9: Effects of $V$ and $p$ on $C_{p, s}\left(N_{C}=5^{2}, N_{T}=3^{2}, N_{p}=3\right.$, and $\left.K=8\right)$

the SDF paging is more likely to find the UE before searching all cells in the residing area. On the other hand, if $\lambda_{m} / \lambda_{c}$ is small, the TAL paging outperforms the movement-based scheme with the SDF paging (in Figure 9 (b), for $V \leq 10 / \lambda_{m}^{2}$, the values of the $\bullet$ curves are larger than 1) because the low-mobility UE is more likely to be found in the last interacted cell, and it is a waste to page all cells in the subarea $A_{0}$. For any $\lambda_{m}$ value, when $V$ increases, more longer $t_{m}$ periods will be observed, and the UE does not move in many consecutive $t_{c}$ periods that fall in these $t_{m}$. In this case, the UE is always found in the last interacted cell, and we observe that $C_{p, T}<C_{p, M}<C_{p, D}$ (in Figures 9 (a) and (b), for $V \geq 2000 / \lambda_{m}^{2}, C_{p, T}<C_{p, M}<C_{p, D}$ ). 
Table 2: The Mobility Management Scheme with the Lowest Paging Cost under Different $\lambda_{m} / \lambda_{c}, V$, and $p$ Values

\begin{tabular}{|c|c|c|c|c|c|c|c|}
\hline \multicolumn{4}{|c|}{ Small $\lambda_{m} / \lambda_{c}$} & \multicolumn{4}{c|}{ Large $\lambda_{m} / \lambda_{c}$} \\
\hline \multicolumn{2}{|c|}{ Small $V$} & \multicolumn{2}{|c|}{ Large $V$} & \multicolumn{2}{c|}{ Small $V$} & \multicolumn{2}{c|}{ Large $V$} \\
\hline Small $p$ & Large $p$ & Small $p$ & Large $p$ & Small $p$ & Large $p$ & Small $p$ & Large $p$ \\
\hline TAL & TAL & TAL & TAL & $\begin{array}{l}\text { Movement } \\
\text { with SDF }\end{array}$ & $\begin{array}{c}\text { Movement } \\
\text { with SDF }\end{array}$ & TAL & TAL \\
\hline
\end{tabular}

From the above discussions, Table 2 lists the mobility management scheme with the lowest paging cost under different input parameter setups. This table shows that the TAL-based scheme outperforms the other schemes when $\lambda_{m} / \lambda_{c}$ is small or $V$ is large.

\section{Conclusions}

This paper studied the LTE TAL-based mobility management scheme by measuring the expected number $C_{u}$ of location updates during the inter-call arrival time and the expected number $C_{p}$ of cells that page the UE when an incoming call arrives. The TAL-based scheme is compared with the movementbased and the distance-based schemes with the SDF paging. We note that the distance-based scheme and the SDF paging cannot be practically implemented, while the TAL-based scheme is a partial implementation of the distance-based scheme with the SDF paging, which can be actually realized. Therefore, the distance-based and the SDF paging schemes are considered as the "optimal performance bounds" for the TAL-based scheme. Surpris- 
ingly, our study indicates that in many scenarios, the TAL-based scheme outperforms the other two schemes, and the details are described as follows.

- For the $C_{u}$ performance, the distance-based scheme outperforms the TAL-based and the movement-based schemes when (1) $p$ is small (i.e., the UE movement exhibits locality) or (2) $\lambda_{m} / \lambda_{c}$ and $V$ are small (i.e., the UE moves infrequently and the movement pattern is regular). On the other hand, the TAL-based scheme has the lowest location update cost when (1) $\lambda_{m} / \lambda_{c}$ and $p$ are large or (2) $V$ and $p$ are large.

- For the $C_{p}$ performance, the movement-based scheme with the SDF paging outperforms the other schemes when $\lambda_{m} / \lambda_{c}$ is large and $V$ is small. For other scenarios (i.e., when $\lambda_{m} / \lambda_{c}$ is small or $V$ is large), the TAL paging outperforms the SDF paging.

As a final remark, the LTE mobility management raises many research issues. In the future, we will study load distribution over multipath network [14], power saving [15], and so on.

\section{Acknowledgements}

R.-H. Liou's work was supported by the MediaTek Fellowship. Y.-B. Lin's work was supported in part by NSC 100-2221-E-009-070 and 101-2221-E-009032, Academia Sinica AS-102-TP-A06, Chunghwa Telecom, IBM, Arcadyan Technology Corporation, ICL/ITRI project under B301EA3300, B301AR2R10, and B352BW1100, Nokia Siemens Networks, Department of Industrial Technology (DoIT) Academic Technology Development Program 100-EC-17-A03-S1-193, and the MoE ATU plan. 


\section{Appendix A. Notation}

The notation used in this paper is listed below.

- $p$ : the probability that the UE moves to the right-hand side neighboring cell

- $\langle x, y\rangle$ : the column and the row labels of an arbitrary cell in a TAL, respectively

- $\langle\bar{x}, \bar{y}\rangle$ : the column and the row labels of the last interacted cell, respectively

- $\left\langle x^{*}, y^{*}\right\rangle$ : the column and the row labels of the cell where the UE currently resides, respectively

- $\langle X, Y\rangle$ : the column and the row labels of an arbitrary TA in a TAL, respectively

- $K$ : the location update threshold for the movement-based and the distance-based schemes

- $A_{j}$ : the subarea $j$ in the SDF paging

- $l_{j}$ : the left-hand side boundary value of $A_{j}$

- $r_{j}$ : the right-hand side boundary value of $A_{j}$

- $C_{u, s}$ : the expected number of location updates between two consecutive incoming calls for the " $s$ " scheme, where $s \in\{T, M, D\}$ denoting the TAL-based, the movement-based, and the distance-based location updates, respectively 
- $C_{p, s}$ : the expected number of cells that page the UE when an incoming call arrives for the TAL paging $(s=T)$, the movement-based scheme with the SDF paging $(s=M)$, and the distance-based scheme with the SDF paging $(s=D)$, respectively

- $T$ : a long observation period

- $t$ : the simulation clock

- $t_{c}$ : the inter-call arrival time

- $t_{m}$ : the inter-move time (i.e., the cell residence time)

- $1 / \lambda_{c}=E\left[t_{c}\right]$ : the mean inter-call arrival time

- $1 / \lambda_{m}=E\left[t_{m}\right]$ : the mean inter-move time

- $V$ : the variance for the $t_{m}$ distribution

- $N_{C}$ : the number of cells in a TA

- $N_{T}$ : the number of TAs in a TAL

- $N_{p}$ : the maximum number of the polling cycles before the UE is found - $n_{a}$ : the number of call arrivals

- $n_{u}$ : the number of location updates

- $n_{p}$ : the number of cells that page the UE when an incoming call arrives

- $n_{C}$ : the number of the inter-call arrival intervals $t_{c}$ in $T$

- $n_{M}$ : the number of the cell crossings in $T$ 
- $n_{m}$ : the number of cell crossings after the last interaction with the network

- $G_{C}$ : the exponential random number generator

- $G_{M}$ : the Gamma random number generator

- $G_{D}$ : the uniform random number generator

- $e, e_{1}, e_{2}$ : the events in the simulation

- $U$ : the uniform random number drawn from $G_{D}$

\section{References}

[1] Yi-Bing Lin and Ai-Chun Pang, Wireless and Mobile All-IP Networks. John Wiley \& Sons, Inc., 2005.

[2] Ian F. Akyildiz, Joseph S. M. Ho, and Yi-Bing Lin, "Movement-based location update and selective paging for PCS networks," IEEE/ACM Transactions on Networking, vol. 4, no. 4, pp. 629-638, August 1996.

[3] Vincent W.-S. Wong and Victor C. M. Leung, "Location management for next-generation personal communications networks," IEEE Network, vol. 14, no. 5, pp. 18-24, September/October 2000.

[4] Shun-Ren Yang, Yung-Chun Lin, and Yi-Bing Lin, "Performance of mobile telecommunications network with overlapping location area configuration," IEEE Transactions on Vehicular Technology, vol. 57, no. 2, pp. 1285-1292, March 2008. 
[5] 3GPP. 3rd Generation Partnership Project; Technical Specification Group Services and System Aspects; General Packet Radio Service (GPRS) enhancements for Evolved Universal Terrestrial Radio Access Network (E-UTRAN) access. Technical Specification 3G TS 23.401 version 10.0.0 (2010-06), 2010.

[6] 3GPP. 3rd Generation Partnership Project; Technical Specification Group Radio Access Network; Evolved Universal Terrestrial Radio Access (E-UTRA) and Evolved Universal Terrestrial Radio Access Network (E-UTRAN); Overall description; Stage 2. Technical Specification 3G TS 36.300 version 10.1 .0 (2010-09), 2010.

[7] Ren-Huang Liou, Yi-Bing Lin, and Shang-Chih Tsai, "An Investigation on LTE mobility management," IEEE Transactions on Mobile Computing, in press, 2011.

[8] Amotz Bar-Noy, Ilan Kessler, and Moshe Sidi, "Mobile users: to update or not to update?," Wireless Networks, vol. 1, no. 2, pp. 175-185, 1995.

[9] Yi-Hua Zhu and Victor C. M. Leung, "Derivation of moving distance distribution to enhance sequential paging in distance-based mobility management for PCS networks," IEEE Transactions on Wireless Communications, vol. 5, no. 11, pp. 3029-3033, November 2006.

[10] Rong Chen, Senmiao Yuan, and Jinquan Zhu, "A dynamic location management method of personal communication system," E-Tech 2004, pp. 1-9, July 2004. 
[11] Ian F. Akyildiz, Janise McNair, Joseph S. M. Ho, Hüseyin Uzunalioğlu, and Wenye Wang, "Mobility management in next-generation wireless systems," Proceedings of the IEEE, vol. 87, no. 8, pp. 1347-1384, August 1999.

[12] F. P. Kelly, Reversibility and Stochastic Networks. New York: Wiley, 1979.

[13] Joseph S. M. Ho and Ian F. Akyildiz, "Mobile user location update and paging under delay constraints," Wireless Networks, vol. 1, no. 4, pp. 413-425, 1995.

[14] Sumet Prabhavat, Hiroki Nishiyama, Nirwan Ansari, and Nei Kato, "Effective Delay-Controlled Load Distribution over Multipath Networks," IEEE Transactions on Parallel and Distributed Systems, vol. 22, no. 10, pp. 1730-1741, October 2011.

[15] Shun-Ren Yang, Sheng-Ying Yan, and Hui-Nien Hung, "Modeling UMTS power saving with bursty packet data traffic," IEEE Transactions on Mobile Computing, vol. 6, no. 12, pp. 1398-1409, December 2007. 